\title{
Variant Alzheimer's Disease with Spastic Paraparesis and Supranuclear Gaze Palsy
}

\author{
Namita Sinha, David Grimes, Shinya Tokuhiro, Christine Sato, Ekaterina Rogaeva, John Woulfe
}

Can J Neurol Sci. 2013; 40: 249-251

Variant Alzheimer's disease (VarAD) is a rare form of familial Alzheimer's disease (AD) and is clinically characterized by progressive dementia which in most cases, is preceded by spastic paraparesis (SP). The clinical diagnosis of VarAD may be difficult in families without a strong family history of dementia and in subjects presenting with paraparesis as the initial symptom.

If VarAD is suspected, the diagnosis can be confirmed by genetic analysis. The presenilin-1 (PSEN1) gene on chromosome $14 \mathrm{q} 24.3$ is the most frequent gene implicated in familial AD, with over 190 different mutations identified to date, some of these are associated with SP (listed at www.molgen.ua. ac.be/ADMutations/). The most common PSEN1 alteration associated with VarAD is an in frame deletion of exon 9 resulting either from a $4.6 \mathrm{~kb}$ genomic deletion or from an exon 9 splice acceptor point mutation. These PSEN1-linked VarAD pedigrees frequently have a distinctive and variant neuropathology, in the form of large, non-cored plaques lacking associated neuritic dystrophy called cotton wool plaques (CWP). These plaques display a distinctive microscopic mass effect whereby they appear to displace adjacent neurons and glial cells.

Several observations argue in favour of the existence of a genetic modifier influencing the phenotype in VarAD cases, including the fact that the identical PSEN1 mutation has been reported in families manifesting either classical or VarAD. ${ }^{1}$ Previously we excluded the possibility that the modifier effect arises from coding variations in three spastic paraparesis genes. ${ }^{2}$ Since then, another gene (ZFYVE26) responsible for spastic paraplegia complicated by mental deterioration was discovered (SPG15 locus on 14q23-24). ${ }^{3}$ ZFYVE26 is a good candidate for the modifier effect because it maps to a locus $\sim 5 \mathrm{Mb}$ upstream from PSEN1, which is notable because there are reports about phenotypic range within a single family (with VarAD and pure AD cases); and such a segregation pattern could be explained if the modifier gene and PSEN1 co-segregated together with a limited number of recombination events.

We describe a case of VarAD with SP and supranuclear gaze palsy with a missense mutation in exon 8 of the PSEN1 gene. Furthermore, we assessed the ZFYVE26 gene as a potential genetic modifier responsible for the variant phenotype.

\section{METHODS}

\section{Immunohistochemistry}

The brain was removed post-mortem and fixed in $20 \%$ neutral buffered formalin for two weeks. Following dissection and gross examination, tissue blocks were selected and embedded in paraffin. Five-micrometer sections were cut and

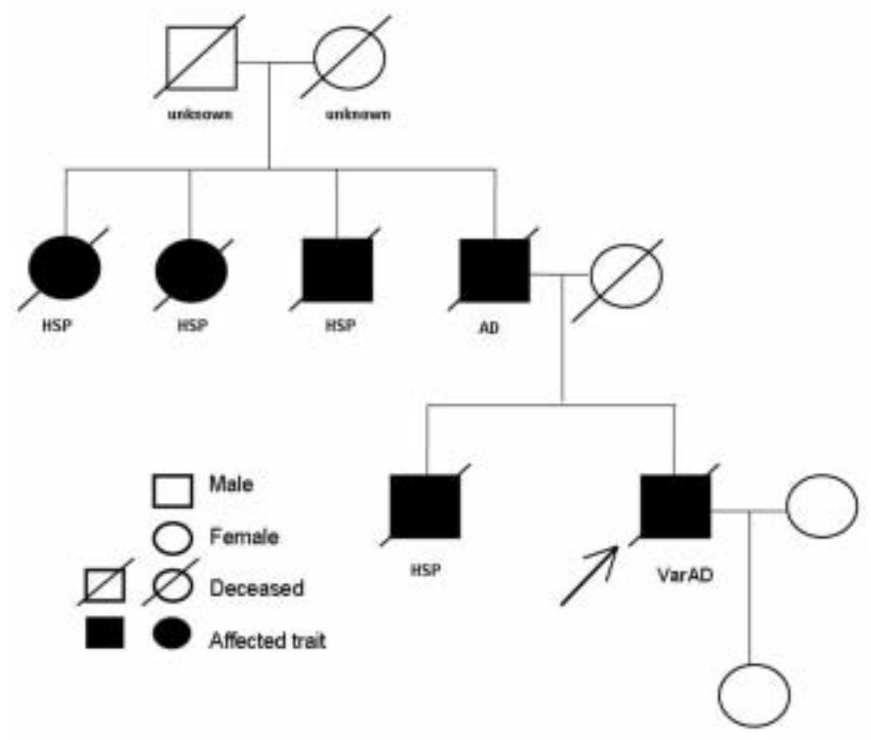

Figure 1: Shows pedigree of family with HSP (Hereditary Spastic Paraparesis, $A D$ (Alzheimer's disease) and varAD (Variant Alzheimer's Disease).

stained with hematoxylin and eosin. Selected sections were also stained using the Bielschowsky silver method and processed immunohistochemically for the detection of tau (Dako, 1:400), ubiquitin (Vector, 1:400), beta-amyloid (Vector, 1:100), TDP-43 (Protein Technology, 1/50), alpha-synuclein (Invitrogen, 1:50) and CD68 (Novocastra, ready to use)

\section{Genetic analysis}

Deoxyribose nucleic acid (DNA) was isolated from frozen brain tissue using a QIAGEN kit. The entire open reading frame of PSEN1 (including exon/intron boundaries) was analyzed in

From the Division of Anatomical Pathology (NS), Queen Elizabeth II Health Sciences Centre and Dalhousie University, Halifax, Nova Scotia; Department of Neurology (DG), Department of Pathology and Laboratory Medicine (JW), Univerity of Ottawa, Ottawa; Center for Research of Neurodegenerative Diseases (ST, CS, ER), Tanz Neuroscience Building, University of Toronto, Toronto, Ontario, Canada.

Received June 14, 2012. Final Revisions Submitted September 7, 2012.

Correspondence to: Namita Sinha, Division of Anatomical Pathology, Queen Elizabeth II Health Sciences Centre and Dalhousie University, Mackenzie Building, 5788 University Avenue, Halifax, Nova Scotia, B3H 1V8, Canada.

Email: drnamitasinha1@gmail.com. 


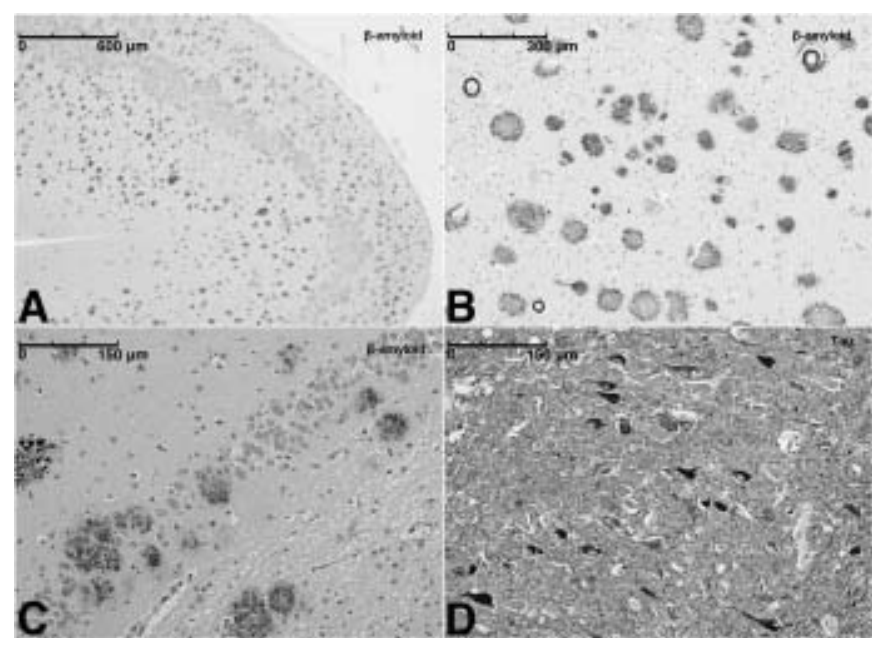

Figure 2: $A \& B$ ) show "Cotton wool plaques" with distinct border, strong positivity to $\beta$-amyloid but only minor neuritic pathology, differentiating them from classical plaques. C) shows the plaques imposing the microscopic mass effect displacing the adjacent neurons and glial cells in hippocampus. D) shows frequent tau-positive neurofibrillary tangles and neuropil threads in frontal cortex.

the patient as described previously. ${ }^{2}$ The mutation analysis of all 42 coding exons of the ZFYVE26 gene was performed as previously described. ${ }^{3}$ Mutations were searched by direct inspection of the fluorescent chromatographs using the SeqScape software (version 1.0) (Applied Biosystems, Foster City, CA).

\section{RESULTS}

\section{Clinical description}

A 66-year-old Caucasian man was brought to the neurology department of our hospital in September 2007 by his cousin. His past history was significant for a diagnosis of polio at the age of five but he did not have any physical limitations throughout his childhood. He had been physically active and involved in sports prior to the onset of pain, weakness and spasticity affecting his legs at age 47. Clinically, primary progressive multiple sclerosis (MS) was initially suspected but all investigations including cerebrospinal fluid analysis, and craniospinal imaging studies were inconclusive. Magnetic resonance imaging (MRI) of head showed mild cerebral atrophy and mild periventricular leukoencephalomalacia. He was noted to "toe walk" and his gait difficulties progressed slowly, to the extent that he required a cane to walk after ten years. At the age of 63 , he was living alone with community support services but was experiencing increasing falls and it was at this point that short term memory difficulties were becoming apparent. He was still able to cook on his own but his family had concerns about his overall safety and after a prolonged hospitalization triggered by a fall, he was transferred to a chronic care facility. In the last two years, his symptoms progressed rapidly. He then required a two person assist for transfers, had marked dysphagia requiring a minced diet with thickened fluids and was incontinent of bladder and bowel. He could recognize family members but could not follow conversations, could not do his finances and was easily confused.

His family history was notable for an elder brother who had a similar disorder starting at age 53 when he first noticed incoordination of his legs and frequent falls during a canoe trip. A few years later, he developed stuttering, and cognitive problems. He died at the age of 60 . No autopsy was performed. The patient's father was diagnosed with $\mathrm{AD}$ and he died in his 50s. His paternal uncle and two paternal aunts had a similar rapidly progressive gait problem (Figure 1 represents pedigree of the patient's family).

On physical examination, he had a marked wide-eyed stare and severe dysarthria making it difficult to understand him. He could not give the date, but knew he was in a hospital although it was the wrong one. He knew his own birth date but could not tell his age. He could not confirm any details of his history accurately and was able to follow only simple one-step commands. He could not perform Luria three-step hand commands. Eye movements were restricted in all directions with marked limitation of upgaze. Saccade speed was slow and he displayed particular difficulty initiating vertical saccades. He appeared to require a blink to initiate a downward saccade. He had a couple of beats of horizontal optokinetic nystagmus but they were absent with the strip moving down. Surprisingly, there was no spasticity or weakness in either upper extremity. However there was marked spasticity in the lower limbs, with only flicker movements distally. He had clonus in the left ankle and bilateral extensor plantar responses. Sensory examination was grossly normal. A diagnosis of autosomal dominant hereditary spastic paraparesis (HSP) with attendant supranuclear gaze palsy was made.

\section{Neuropathology Gross}

The patient died at the age of 67 and the autopsy was limited to the brain. The right frontal pole was frozen and stored at -70C. The brain weighed $1,110 \mathrm{~g}$ after fixation. Gross examination showed moderate diffuse atrophy of the cortex and hippocampus with bilaterally symmetric hydrocephalus ex vacuo. The substantia nigra and locus coeruleus were normally pigmented. The medullary pyramids appeared greyish in colour.

\section{Microscopic}

Histopathologic examination revealed frequent numerous CWP in all cerebral lobes, basal ganglia, limbic areas and brainstem which immunostained intensely for beta- amyloid (Figure 2A, 2B and 2C). The cotton wool plaques were unusually large, often exceeding $100 \mu \mathrm{m}$ in diameter, with distinct borders but devoid of an eosinophilic core. As has been described previously for CWP, they imposed microscopic "mass effect" and appeared to displace adjacent neurons and glial cells (Figure 2C). Beta-amyloid deposits were also present in the walls of cortical and leptomeningeal blood vessels as well as within capillaries in the white matter. In addition to these betaamyloid deposits, there were frequent tau-positive neurofibrillary tangles and neuropil threads in the cerebral cortex, entorhinal cortex and hippocampus (Figure 2D). No TDP-43- or alpha-synuclein- immunoreactive intraneuronal or glial inclusions were observed. 
In the context of the clinical presentation, the histopathological findings were highly suggestive of VarAD with SP. Consequently, on the basis of the morphological findings, tissue was sent for genetic analysis; specifically for detection of a PSEN1 mutation.

\section{Genetic test results}

The study was conducted in accordance with the University of Toronto ethical review board. Sequencing of the entire open reading frame of the $P S E N 1$ gene revealed a heterozygous $A>G$ transition (GAA to GGA) in exon 8, leading to an amino acid substitution of Glu (E) to Gly (G) at codon 280 (g.50024A>G relative to Met1 in AF109907.1). This E280G substitution affects a highly conserved codon in the HL-VI region of the protein. The pathogenicity of the E280G mutation is indisputable as it has previously been reported as an AD-causing substitution in six independent publications (www.molgen.ua.ac.be/ADMutations/).

The analysis of the ZFYVE26 gene did not reveal a truncating mutation, however we detected two known heterozygous missense variations: the N1891S (rs3742883) and T898S (rs17192170) with reported frequencies in European population ranging between $4 \%$ and $7 \%$ (http://www.ncbi.nlm.nih.gov/ genome/ guide/human/).

\section{DisCUSSION}

The earliest description of the combination of dominantly inherited Alzheimer-type presenile dementia and spastic paraparesis was published in 1940 but no genetic analysis was available. Since that time VarAD has been known as a rare familial neurodegenerative disease defined clinically by dementia and SP; pathologically by CWPs, and genetically by mutations in PSEN1.

Variant Alzheimer's disease has been reported in Finnish, Australian, British, Belgian and Japanese families and the PSEN1 mutations in these pedigrees most frequently involve exon 9 (www.molgen.ua.ac.be/ADMutations). This study is the first to demonstrate autopsy-confirmed AD with SP and supranuclear gaze plasy due to E280G mutation in the PSEN-1 gene. This mutation was first identified in an Irish family with one of the nine affected siblings having CWP pathology. ${ }^{4}$ Supranuclear gaze palsy was not reported in any of the family members. The supranuclear gaze palsy in our case was unusual and included particular difficulty in initiating vertical saccades, suggesting an apraxia. Vertical gaze palsies are rarely associated with HSP but are the hallmark of the hereditary spastic ataxia SAX-1 disease. ${ }^{5}$ Hereditary spastic paraparesis is a heterogeneous group of disorders characterised by slowly progressive bilateral lower limb spasticity. Although SP is a main feature of this condition, it has an earlier age of onset, a slow progression over decades, and dementia is not a prominent feature.

Variant Alzheimer's disease is a neurodegenerative disease that is likely underdiagnosed. Dementia can occur very late in the disease course and a wide variety of associated neurologic features can be present including supranuclear gaze palsy. Many of the clinical and pathologic features are unique yet poorly understood. Indeed, in the present case, the unique histopathology provided the impetus to pursue genetic testing. It has been suggested that for these individuals a gene modifier is present yet its identification remains elusive.

\section{Conclusion}

Our analysis of the ZFYVE26 gene did not reveal truncating mutations (the type of mutations reported to cause spastic paraplegia in HSP15-linked families). ${ }^{3}$ However, it would be important to verify the pathological significance of two missense variations (rs3742883 and rs17192170) detected in our patient and assess the presence of these substitutions in other patients with VarAD.

\section{ACKNOWLEDGMENTS}

This work was supported by grants from the Canadian Institutes of Health Research, Weston Foundation, the Canadian Institutes of Health Research, Ontario Research Fund (ER).

\section{REFERENCES}

1. Rogaeva E. The solved and unsolved mysteries of the genetics of early-onset Alzheimer's disease. Neuromol Med. 2002;2(1): $1-10$.

2. Rogaeva E, Bergeron C, Sato C, et al. PS1 Alzheimer's disease family with spastic paraplegia: the search for a gene modifier. Neurology. 2003;61(7):1005-7.

3. Hanein S, Martin E, Boukhris A, et al. Identification of the SPG15 gene, encoding spastizin, as a frequent cause of complicated autosomal-recessive spastic paraplegia, including Kjellin syndrome. Am J Hum Genet. 2008;82(4):992-1002.

4. O'Riordan S, McMonagle P, Janssen JC, et al. Presenilin-1 mutation (E280G), spastic paraparesis, and cranial MRI whitematter abnormalities. Neurology. 2002;59(7):1108-10

5. Grewal KK, Stefanelli MG, Meijer IA, Hand CK, Rouleau GA, Ives EJ. A founder effect in three large Newfoundland families with a novel clinically variable spastic ataxia and supranuclear gaze palsy. Am J Med Genet A. 2004;131(3):249-54. 